\title{
Kinetic occlusion: Further studies of the boundary-flow cue
}

\author{
LINCOLN G. CRATON and ALBERT YONAS \\ University of Minnesota, Minneapolis, Minnesota
}

\begin{abstract}
Previous work has demonstrated that human beings employ a processing assumption, the boundary-flow constraint, in perceiving the order of depth at an edge. Subjects perceive depth order of surfaces on the basis of the relative motions of an image boundary and a projected surface texture on either side of the boundary. In the present study, adult subjects viewed computergenerated kinematograms in which boundary-flow information provided the only cue for depth order. The results of Experiments 1 and 2 indicate that common motion between boundary and texture and differential motion between boundary and texture can independently generate the perception of ordered depths of surfaces. In Experiment 3, we examined the interaction of two processes involved in the extraction of depth order from boundary-flow displays: (1) the propagation of foreground and background surfaces from texture to boundary; and (2) the computation of depth order of surfaces on either side of the boundary. The results indicate that while the mechanism that computes depth from boundary-flow information functions reliably when the mean dis-

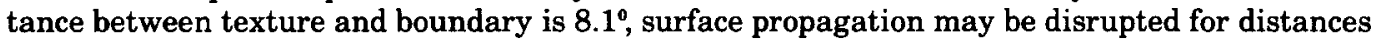
of this magnitude.
\end{abstract}

To distinguish foreground from background in the visual field is a fundamental task for any visually sensitive organism. One way in which this task might be accomplished would be through the detection of lawful patterns of change in the retinal image resulting from motion of the observer and/or objects. For instance, as an observer moves about in an environment, some surfaces are progressively occluded from the observer's view and others are disoccluded. Until recently, it was thought that human perception of the kinetic occlusion of one surface by another was due to the appearance (accretion) and disappearance (deletion) of surface texture that occurs when the occluded surface is progressively uncovered or covered, respectively (Gibson, Kaplan, Reynolds, \& Wheeler, 1969; Kaplan, 1969). Kaplan (1969) presented adult subjects with a film consisting of a rectangular area filled with randomly located texture elements. When this display was stationary, it appeared to be a single continuous textured surface. When the texture underwent lateral motion, a subjective contour was perceived at the margin where texture elements were accreted and deleted. The subjects reported a vivid impression of relative depth at the contour, with the accreted and deleted texture ap-

This research was supported by a National Institute of Child Health and Human Development grant awarded to Albert Yonas (HD-16924). The first author was supported by a Graduate Fellowship to the Center for Research in Learning, Perception, and Cognition from the National Institute of Child Health and Human Development (HD-07151-07). The authors wish to thank Kim Pearson and Ann Bensen for their valuable assistance, and Jonathon Marshall and two anonymous reviewers for comments on earlier drafts. Correspondence and requests for reprints should be sent to Lincoln G. Craton, Department of Psychology, University of Illinois, $603 \mathrm{E}$. Daniel St., Champaign, IL 61820. pearing as part of an occluded surface. More recent studies have yielded similar results with adults (Andersen \& Braunstein, 1983) and 5-month-old infants (Granrud et al., 1984).

Thompson, Mutch, and Berzins (1985) have shown that there is an alternative source of information for the order of depth at an edge. The relative depth order of surfaces is specified by the relationship between the motion of a projected depth edge, referred to here as an image boundary, and that of projected texture elements from the two surfaces on either side of the boundary. The principle underlying this account is that an image boundary moves with the image of the occluding surface to which it belongs. We define boundary flow to be the image-plane velocity of a projected edge; surface flow refers here to the image-plane velocity of texture elements on either side of the boundary. The boundary-flow constraint holds that when a rigid surface covers or uncovers a second surface at an edge, the boundary flow is the same as the surface flow of the occluding surface at the boundary. More specifically, the image-plane velocity of projected surface elements from an occluding surface approaches the velocity of the image boundary as a mathematical limit. Thus, the constraint applies to projected surface texture immediately adjacent to the boundary. Any image region not satisfying the boundary-flow constraint cannot correspond to an occluding surface in the scene. 1

Both Kaplan's (1969) displays and those used in more recent studies of kinetic occlusion contained boundaryflow information for depth in addition to accretion and deletion information. In order to demonstrate human sensitivity to boundary-flow information independent of accretion/deletion information, we (Yonas, Craton, \& 
Thompson, 1987) presented adult subjects with randomdot kinematograms in which two separated random-dot regions moved with respect to one another (see Figure 1). The texture regions moved in counterphase, alternately toward and away from the center of the display. A vertical boundary was placed between the dot regions. The boundary was composed of either a vertical line (objective contour condition, see Figure 1A) or a subjective contour (subjective contour condition, see Figure 1B). This edge representation moved rigidly with the dot region on either the left or the right. An area of blank space between the boundary and the texture region moving in counterphase to the boundary eliminated depth information from the accretion and deletion of texture elements.

In addition to manipulating the information specifying a depth edge, the width of the "gap" between boundary and texture regions for displays in the objective contour condition was varied to produce small, medium, and large gap conditions. In the subjective contour condition, only the small gap version was presented. Accretion/deletion displays comparable to those used by Kaplan (1969) were also presented. In these displays, the direction and velocity of motion of the boundary and texture elements were

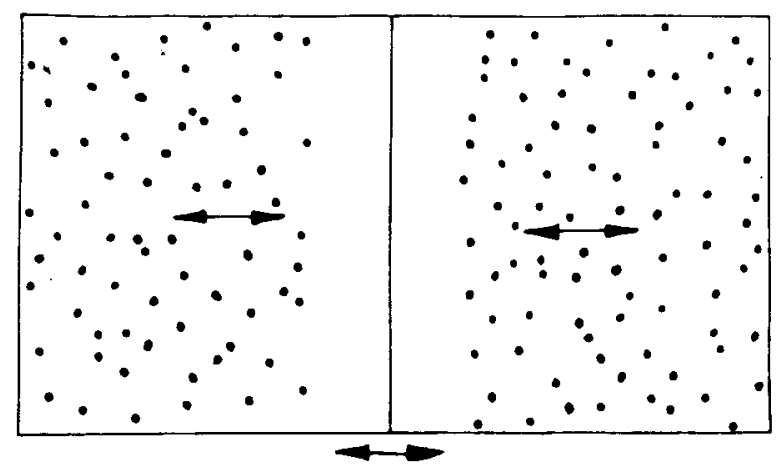

(a)

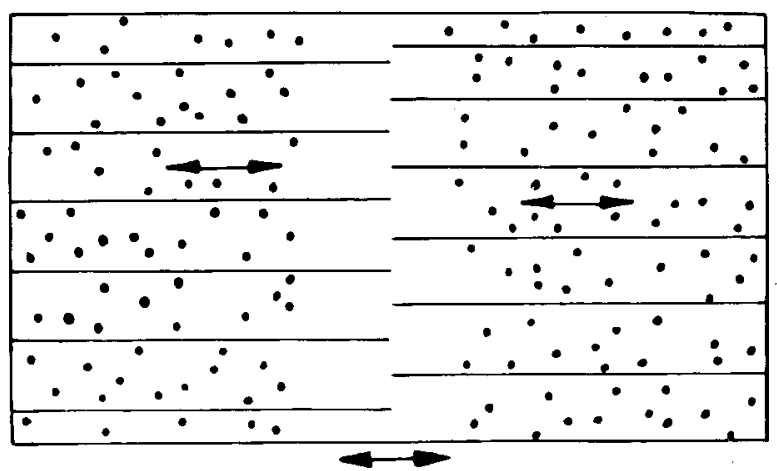

(b)

Figure 1. Schematic drawing of boundary-flow displays. Arrows indicate motion of the texture fields and central contour. A "gap" between the central edge representation and texture elements eliminates accretion and deletion information for depth order. (A) In the objective contour condition, a vertical line provides information for a depth edge. (B) In the subjective contour condition, endstopped horizontal lines generate a vertical subjective contour. identical to those in the boundary-flow conditions, except that the texture fields extended all the way to the boundary. As a result, texture was progressively accreted and deleted on the side that moved differently than the boundary, and texture was preserved (i.e, not accreted and deleted) on the side that moved identically with the boundary.

Both the small gap boundary-flow conditions and accretion/deletion displays yielded high frequencies of depth judgments in the predicted direction; performance in these conditions did not differ significantly. When viewing small gap displays with either an objective or a subjective contour, subjects reported perceiving a continuous foreground surface connecting the boundary to the dot region that moved with it. The boundary was perceived as the edge of this surface. The dots on the other side, which moved in a direction opposite to the motion of the boundary, were seen as belonging to a background surface that extended behind the boundary. This background surface was perceived as being covered and uncovered by the foreground surface. More recent work has extended this result to 5-month-old infants (Craton \& Yonas, 1988). An additional finding in the adult study was that the frequency of depth judgments in the predicted direction diminished as the widths of the gaps in boundaryflow displays increased.

Because human sensitivity to boundary-flow information is a recent discovery, very little is known about the mechanisms that employ this constraint. Our primary goal in the experiments reported below was to specify more precisely the kinetic stimulus variables that determine the perception of depth at an edge from boundary-flow information. One question concerns whether information relating boundary flow to surface flow on both sides of the boundary is required for obtaining a depth effect, or whether surface flow from only one surface is sufficient. Logically, there are two potential sources of information for relative depth order based on the relation of boundary flow and surface flow: (1) the common motion of texture and boundary that specifies an occluding surface; and (2) the differential motion of texture and boundary that specifies an occluded surface.

The first of these relations can be viewed as an elaboration of the Gestalt principle of "common fate" (Wertheimer, 1923). This principle holds that elements which move simultaneously in the same direction are readily grouped into perceptual units. Such units tend to appear as shaped figures in front of a formless ground. In the present formulation, one of the moving elements is an image boundary corresponding to a depth edge. The second relation above points to the fact that the lack of common fate between projected surface texture and an image boundary also constitutes information for the depth order of surfaces. When a rigid surface occludes a second surface, the boundary flow and the motion of projected surface elements of the occluded surface immediately adjacent to the boundary are different.

In Experiment 1, adult subjects viewed kinetic displays in four conditions. In two of the conditions, a single dot region, on either the right or the left side of the bound- 


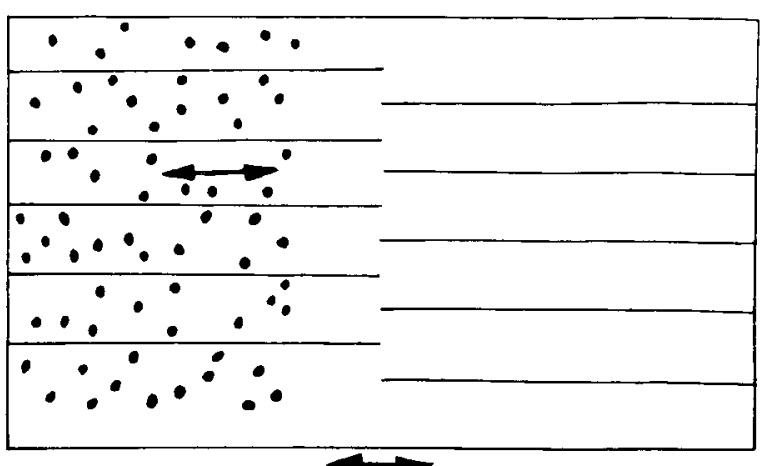

(a)

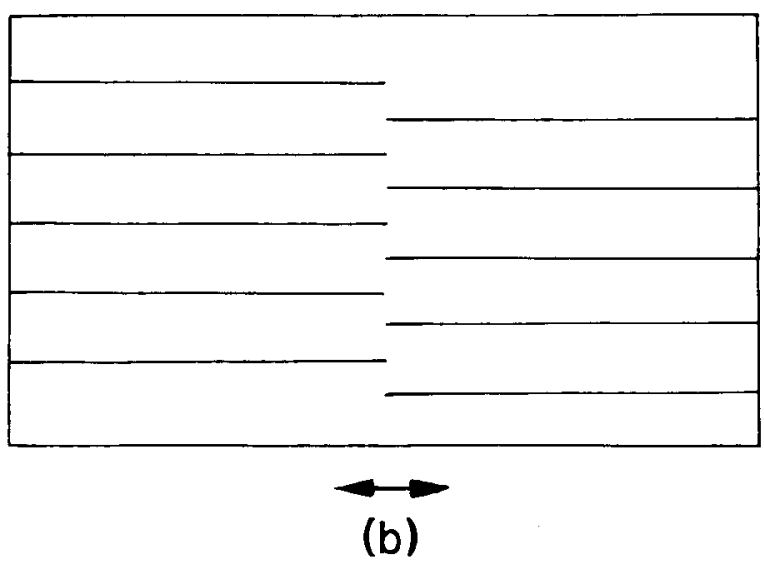

Figure 2. Schematic drawing of displays used in Experiment 1. Arrows indicate motion of the texture field and central contour. (A) Texture elements on one side of the subjective contour moved either rigidly with the contour (common motion condition) or in counterphase motion with the contour (differential motion condition). (B) No-texture control display.

ary, was present (Figure 2A). This region moved either rigidly with the boundary (common motion condition) or in counterphase motion with the boundary (differential motion condition). A third display condition (common motion and differential motion combined) contained dot regions on both sides of the boundary (see Figure 1B); one of the dot regions moved rigidly with the boundary, while the other simultaneously moved in counterphase motion with the boundary. In the fourth condition (no texture control), texture was absent on both sides of the boundary; only end-stopped horizontal lines, used to generate the subjective contour, were present (Figure 2B).

\section{EXPERIMENT 1}

\section{Method}

Subjects. Fourteen unpaid students at the University of Minnesota participated in the experiment as part of the requirements of an introductory psychology course.

Apparatus. Stimuli were presented on a CRT, refreshed every $5 \mathrm{msec}$ by an IBM AT microcomputer. The displays were observed through an $8.5-\mathrm{cm}$ high and $15.5-\mathrm{cm}$ wide aperture (viewing distance: $70 \mathrm{~cm}$; visual angle: $6.9^{\circ}$ vertically, $12.5^{\circ}$ horizontally) attached to the face of the CRT. Phosphor persistence was minimal and did not result in excessive streaking from the translating dots. An eyepatch worn over one eye eliminated binocular information. A keyboard was located directly in front of the subject. A shaded light provided dim overall illumination for the experimental room, sufficient for the dark-adapted subjects to see the response keys on the keyboard.

Displays. Four display conditions were presented. In each of the four conditions, a vertical subjective contour was displayed at the center of the screen. The subjective contour was created by a set of 10 horizontal lines that extended behind the viewing aperture on either side of the display and ended at the same vertical midline of the display (Figures 1B and 2). These horizontal lines provided information for the presence of surfaces on both sides of the contour. Horizontal movements of the subjective contour were generated by simultaneously lengthening the horizontal lines on one side of the display and shortening the horizontal lines on the other side. The subjective contour translated back and forth continuously (angular displacement, $1.6 \% \mathrm{sec}$ ), reversing direction approximately every $1 \mathrm{sec}$. For displays containing random texture elements, accretion and deletion of some elements occurred at the right and left borders of the viewing aperture. The four display conditions were as follows:

Common and differential motion combined. In this condition, randomly distributed dots to the left and right of the contour formed two texture fields, each containing approximately 80 dots (visual angle of each dot: $1^{\circ}$ ). Both texture fields were separated from the central contour by blank space. These textureless gaps allowed for lateral motion of the contour and texture fields without providing accretion and deletion cues for depth order. All the dots in a texture field moved in synchronous horizontal motion (angular displacement, $1.6^{\circ} / \mathrm{sec}$ ). The two texture fields alternately approached and receded from one another, reversing direction approximately every $1 \mathrm{sec}$. On each trial, the lateral motion of the centrally located vertical contour was identical to that of one of the two texture fields; the contour thus moved in counterphase motion with texture on the opposite side. Consequently, both the common motion of a boundary (specified by the subjective contour) and texture on one side and the differential motion of the boundary and texture on the other side provided information for the relative depth of surfaces in this condition. The width of the gap between the contour and identically moving texture field was constant $\left(.8^{\circ}\right)$. The gap width between the contour and the texture field not tied to the motion of the contour changed as the two sides of the display approached and receded from one another (range: $.1^{\circ}-1.6^{\circ}$ ). Thus, the average width of the varying gap was equal to the width of the constant gap.

Common motion condition. In this condition, the common motion of a texture field and boundary provided the only information for depth order of surfaces. A single texture field moved rigidly with the subjective contour (Figure 2A). The motion parameters and constant gap size were the same as that for the contour and identically moving texture field in the common and differential motion combined condition. Thus, displays in this condition were identical to the common and differential motion combined condition except that they lacked a texture field whose motion differed from that of the contour.

Differential motion condition. In this condition, differential motion of a texture field and boundary provided the only information for the order of depth at an edge. Displays in this condition were identical to those in the common and differential motion combined condition, except that they lacked a texture field that moved identically with the subjective contour.

No texture condition. In this control condition, no information was available for the depth order of surfaces from the relative motion of texture and boundary. Displays in this condition contained only a moving subjective contour produced by horizontal inducing lines. One can observe that the static version of this display sometimes produces the perception of two surfaces ordered in depth (Figure 2B). 
However, this depth ordering is bistable; that is, it alternates spontaneously. We investigated the frequency with which kinetic versions of this display also produced bistable depth perception.

Procedure. Before being tested, the subjects were given the following instructions: "Look at the display and decide which of the following is true: (1) the left side looks like it is in front; (2) the right side looks like it is in front; (3) both sides appear to be the same distance away."

The subjects were instructed to record their responses by pressing one of three keys on a keyboard directly in front of them. Each subject received three practice trials in which two sheets of paper were held in front of the CRT by the experimenter. The relative depth of the sheets of paper was varied so that the one on the left was closer to the subject on one trial, the one on the right was closer to the subject on one trial, and the sheets were equidistant from the subject on one trial. Practice trials were presented in random order, and the subjects were asked to indicate which button represented the appropriate response. All subjects performed quickly and without error on the practice trials.

During test trials, the subjects observed displays monocularly from a distance of $70 \mathrm{~cm}$. Head movements were not restricted. The subjects viewed a total of 200 trials, 50 from each of the display conditions. A new random distribution of texture elements was generated for each trial. Displays were generated in blocks of eight trials, such that each display type occurred twice in each block. Within each block, the predicted depth orderings for displays in the three experimental conditions were counterbalanced, so that for each display condition there was one trial specifying an occluding surface on the left and another trial specifying an occluded surface on the right. The order of presentation within blocks was random. Every display continued without interruption until the subject recorded a response. After each response, there was a brief pause, and then the next display was generated. Total testing time was approximately $45 \mathrm{~min}$.

\section{Results and Discussion}

The mean percentages of depth judgments consistent with predicted depth order and opposite predicted depth order, as well as mean percentages of "no depth" responses, are presented in Table 1. The main purpose of Experiment 1 was to determine whether adults are sensitive to depth information specified by two types of relation between the optical motion of a boundary and the optical motion of texture elements adjacent to the boundary. As the data in Table 1 show, adults perceived depth at an edge on the basis of the common motion of texture and a boundary and differential motion between texture and boundary, when these cues were isolated in kinetic displays. In addition, the depth effect obtained for the common and differential motion combined condition (see Table 1) replicates previous studies indicating that adults and 5-month-old infants perceive depth at an edge when both the common motion of boundary and texture on one side and the differential motion of boundary and texture on the other side are present.

After each experimental session was completed, the observers were asked to describe their strategies for making depth judgments. All of the subjects maintained that their responses were based on the phenomenal impression of ordered depth of surfaces in three-dimensional space, and it was clear from their descriptions that their ability to perform the task did not involve conscious reasoning about the relations between the motion of the subjective contour and texture elements. The observers were also asked to estimate the perceived separation in depth of the two surfaces. Most subjects commented that it was hard to ascertain any precise amount of depth separation, although several observers reported that the foreground side of the display appeared to "slide" over the background side such that the foreground surface seemed to be in contact with the background surface.

An unexpected finding was that the differential motion displays were more effective than the common motion displays in generating the predicted depth effect. A one-way ANOVA carried out on the mean number of responses consistent with predicted depth order for the three experimental conditions yielded a significant main effect for condition $[F(2,26)=12.67, p<.001]$. Tukey post hoc comparisons revealed that the common motion condition generated significantly fewer responses in the predicted direction than did either the differential motion condition or the common and differential motion combined condition $(p<.05)$. Inspection of the individual data showed that 11 out of the 14 subjects responded with the predicted depth order more frequently in the differential motion condition than in the common motion condition. The remaining 3 subjects responded as predicted more often in the common motion condition. A sign test confirmed that the number of subjects showing higher frequency of predicted responding was significantly greater in the differential motion condition $(p<.05)$. A second one-way ANOVA carried out on the mean number of "no depth" responses for each of the four display conditions revealed a significant main effect for condition $[F(3,39)=17.38$, $p<.001]$. Post hoc comparisons based on Tukey's honestly significant difference method indicated that the differential motion condition and the common and differential motion combined condition yielded signifi-

Table 1

Mean Percentages of Depth Order Judgments, with Standard Deviations: Experiment 1

\begin{tabular}{|c|c|c|c|c|c|c|}
\hline \multirow[b]{3}{*}{ Motion Condition } & \multicolumn{6}{|c|}{ Percent Response } \\
\hline & \multicolumn{2}{|c|}{$\begin{array}{c}\text { Predicted } \\
\text { Depth Order }\end{array}$} & \multicolumn{2}{|c|}{ No Depth } & \multicolumn{2}{|c|}{$\begin{array}{l}\text { Opposite Predicted } \\
\text { Depth Order } \\
\end{array}$} \\
\hline & $M$ & $S D$ & $M$ & $S D$ & $M$ & $S D$ \\
\hline Common and differential combined & 97.4 & 5.5 & 0.9 & 2.7 & 1.6 & 4.8 \\
\hline Common & 69.6 & 25.0 & 26.7 & 25.9 & 3.7 & 6.5 \\
\hline Differential & 91.7 & 7.9 & 6.1 & 6.86 & 2.1 & 4.0 \\
\hline Control & & & 42.6 & 31.1 & & \\
\hline
\end{tabular}


cantly fewer responses of "no depth" than did the notexture control condition. The common motion condition, on the other hand, did not differ from the no-texture control condition in the number of "no depth" responses. The common motion condition produced significantly more "no depth" responses than did either of the other two experimental conditions $(p<.05)$.

The results of Experiment 1 suggest that information about the differential motion of texture and a boundary is more effective than information about the common motion of boundary and texture in generating the perception of depth at an edge. In Experiment 2, we examined an alternative explanation for this finding, based on differences in the size of the gaps between texture and contour in the common motion and differential motion displays. These gaps were necessary in order to eliminate depth information from the accretion and deletion of texture elements. While the average width of the gap between the subjective contour and texture in the differential motion condition was equal to the constant gap width in the common motion condition $\left(8^{\circ}\right)$, the gap width in the differential motion condition varied from $.1^{\circ}$ to $1.6^{\circ}$ visual angle. Thus, the distance between contour and texture was smaller in the differential motion condition than in the common motion condition for approximately half the duration of each trial. The texture was immediately adjacent to the contour each time it approached the differently moving contour, before both the texture and the contour changed direction.

Experiment 2 was designed to test the hypothesis that the superiority of the differential motion condition in Experiment 1 was due to the smaller minimum retinal distance between the subjective contour and texture in this condition. This hypothesis predicts that when the constant gap width in the common motion condition equals the minimum gap width in the differential motion condition, there should be no differences between the two conditions in the frequency of predicted depth responses.

\section{EXPERIMENT 2}

\section{Method}

Subjects. Seventeen unpaid subjects at the University of Minnesota participated in the experiment as part of the requirements of an introductory psychology course.

Apparatus. The displays were generated by a Commodore Amiga microcomputer and observed through a 9-cm high and $17.7-\mathrm{cm}$ wide aperture (viewing distance: $35 \mathrm{~cm}$; visual angle: $14.4^{\circ}$ vertically, $26.8^{\circ}$ horizontally). In all other aspects, the apparatus was identical to that used in Experiment 1.

Displays. Six display conditions were presented. The five experimental conditions were identical to the common motion and differential motion conditions used in Experiment 1, except that the width of the gap between contour and texture was varied within both of these conditions. The width of the constant gap in the common motion displays was varied to produce small constant gap, medium constant gap, and large constant gap conditions $\left(.1^{\circ}, 1.6^{\circ}\right.$, and $3.3^{\circ}$ visual angles, respectively). The average width of the varying gap in the differential motion displays was also varied to produce small varying gap and large varying gap conditions (ranges: $.1^{\circ}-3.3^{\circ}$ and $1.6^{\circ}-5.0^{\circ}$, respectively). The subjective contour and texture fields translated at $1.6 \% \mathrm{sec}$, reversing direction every $1 \mathrm{sec}$. The no-texture control condition used in Experiment 1 was also presented.

Procedure. The general procedure was equivalent to that used in Experiment 1, except that subjects viewed a total of 180 trials, 20 in each of the six display conditions. Total testing time was approximately $35 \mathrm{~min}$.

\section{Results and Discussion}

Table 2 shows the mean percentages of depth judgments consistent with predicted depth order and opposite predicted depth order, as well as the mean percentages of "no depth" responses. A one-way repeated measures ANOVA conducted on the mean number of responses consistent with predicted depth order revealed a significant main effect for condition $[F(4,64)=3.65, p<.01]$. Four planned comparisons each revealed significant differences $(p<.05)$, discussed below.

The data in Table 2 replicate several findings from Experiment 1 . First, both the common motion and differen-

Table 2

Mean Percentages of Depth Order Judgments, with Standard Deviations: Experiment 2

\begin{tabular}{|c|c|c|c|c|c|c|}
\hline \multirow[b]{3}{*}{ Gap Size } & \multicolumn{6}{|c|}{ Percent Response } \\
\hline & \multicolumn{2}{|c|}{$\begin{array}{c}\text { Predicted } \\
\text { Depth Order }\end{array}$} & \multicolumn{2}{|c|}{ No Depth } & \multicolumn{2}{|c|}{$\begin{array}{l}\text { Opposite Predicted } \\
\text { Depth Order }\end{array}$} \\
\hline & $M$ & $S D$ & $M$ & $S D$ & $M$ & $S D$ \\
\hline \multicolumn{7}{|c|}{ Differential Motion Condition } \\
\hline Small varying gap & 89.7 & 11.8 & 8.0 & 9.5 & 2.4 & 4.7 \\
\hline Large varying gap & 90.0 & 10.6 & 7.4 & 8.5 & 2.6 & 4.4 \\
\hline \multicolumn{7}{|c|}{ Common Motion Condition } \\
\hline Small constant gap & 80.0 & 20.5 & 14.4 & 14.2 & 5.6 & 15.0 \\
\hline Medium constant gap & 76.5 & 21.6 & 16.8 & 17.0 & 6.8 & 18.4 \\
\hline Large constant gap & 76.2 & 24.6 & 17.1 & 17.4 & 6.8 & 19.6 \\
\hline \multicolumn{7}{|c|}{ Control } \\
\hline & & & 37.4 & 28.4 & & \\
\hline
\end{tabular}

Note-Small and large varying gaps were $.1^{\circ}-3.3^{\circ}$ and $1.6^{\circ}-5.0^{\circ}$ visual angle, respectively. Small, medium, and large constant gaps were $.1^{\circ}, 1.6^{\circ}$, and $3.3^{\circ}$ visual angle, respectively. 
tial motion conditions generated the predicted depth effect. The present results also replicate the finding that when the gap width in common motion displays equals the average gap width in differential motion displays, the differential motion displays are more effective in producing a depth effect. The small varying gap $\left(.1^{\circ}-3.3^{\circ}\right)$ condition generated significantly more responses with the predicted depth order than did the medium constant gap $\left(1.6^{\circ}\right)$ condition $[t(16)=2.38, p<.05]$. Similarly, the large varying gap condition $\left(1.6^{\circ}-5.0^{\circ}\right)$ generated significantly more responses than did the large constant gap $\left(3.3^{\circ}\right)$ condition $[t(16)=2.15, p<.05]$.

The data do not support the hypothesis that the minimum retinal distance between boundary and texture accounts for the superiority of the differential motion condition over the common motion condition in generating perceived depth from boundary-flow displays. The percentages of depth responses in the predicted direction for the small varying gap condition and the large varying gap condition were nearly identical; the depth judgments in the small, medium, and large constant gap conditions were also quite comparable (see Table 2). At the same time, the results of two planned comparisons indicate that both of the varying gap conditions produced significantly more depth responses in the predicted direction than did the constant gap conditions containing identical minimum gap, sizes [small varying gap condition, $t(16)=1.78$, $p<.05$; large varying gap condition, $t(16)=2.50$, $p<.05]$.

Note that the no-texture control condition in Experiments 1 and 2 generated the perception of surfaces ordered in depth $(57.4 \%$ and $62.6 \%$ of the time, respectively), despite the fact that no information for a particular depth ordering was available. This result is not surprising, given the bistable depth percept that is sometimes generated by the static version of this display (Figure 2B). The perception of "unsigned" depth in both the static and the kinetic cases may be due to the presence of T-shaped intersection features formed by the intersection of the subjective contour with the horizontal lines used to induce it. The (normally) vertical segment or stem of a $T$ intersection cannot be closer to the viewer than the segment forming the top of the T (Binford, 1981). Figure 2B contains some $T$ intersections with the stem of the $T$ located on the right of, and others with the stem on the left of, the top of the T specified by the subjective contour. Consequently, there is conflicting information for the depth order of the right and left surfaces, resulting in the spontaneous reversal of perceived depth order that occurs with prolonged viewing. When boundary-flow information is added to these displays, the percept changes in two ways: First, the frequency of perceived depth increases substantially; and second, the perceived depth ordering becomes stable (see Table 1).

Taken together, Experiments 1 and 2 provide convincing evidence for human sensitivity to the two components of boundary-flow information for the order of depth at an edge: (1) the common motion of projected surface tex- ture and an image boundary that specifies an occluding surface; and (2) the differential motion of texture and boundary that specifies an occluded surface. One implication of this result is that the boundary-flow cue is not reducible to the Gestalt principle of "common fate." This principle is consistent with the depth effects obtained in the common motion condition. However, results for the differential condition indicate that the lack of common fate between a boundary and texture on one side of the boundary provides the visual system with information for the depth order of surfaces. Texture that moves inconsistently with a boundary is perceived as a surface that is being occluded by another surface whose edge is specified by the boundary. In fact, when these two kinetic cues are isolated, the "uncommon fate" of a boundary and texture appears to produce a more vivid impression of depth than does the common fate of boundary and texture.

\section{EXPERIMENT 3}

In Experiment 3, we investigated the local versus global processing characteristics of the mechanism that extracts depth from boundary-flow information. Thompson et al. (1985) have shown that algorithms which measure local surface flow (i.e., flow occurring immediately adjacent to the boundary) can exploit the boundary-flow constraint in order to recover depth order for a variety of types of object motion. In certain cases, such as when an object is rotating around an axis parallel to the image plane (rotation in depth), local measurements of surface flow are required for veridical depth perception. Consider the parallel projection of a textured opaque cylinder that is rotating about a fixed vertical axis and is presented against a homogenous background. The projected texture undergoes a sinusoidal shift in velocity, such that the velocity of a given texture element approaches that of the two stationary vertical boundaries as the distance between texture and a boundary is reduced. Local measurements of surface flow could in principle be used to determine that the texture and boundaries are part of the same foreground object. In contrast, a mechanism that measured surface flow some distance from the boundaries might mistake this stimulus for an occluded surface passing behind an invisible aperture, since surface flow in the center of the visible surface of the cylinder is quite different from boundary flow.

In the case of parallel projection of a rigid object translating in a direction parallel to the image plane (translation at constant depth), boundary flow will equal surface flow measured in any region of the image corresponding to the occluding surface. Consequently, measurements of surface flow taken quite far away from image boundaries can in principle be used to extract the depth order of surfaces.

Is the human visual system limited to local measurements of surface flow for computing depth order from boundary-flow information? Yonas et al. (1987) found that for translation motion, the depth effect produced by 
boundary-flow displays diminishes as the width of the gap between texture and a boundary is increased. Subjects viewing displays in a large gap condition, in which the separation between a boundary (specified in this case by a vertical line, see Figure 1A) and identically moving texture was $3.1^{\circ}$ and the separation between boundary and differently moving texture ranged from $2.5^{\circ}$ to $3.8^{\circ}$, reported perceiving depth in the predicted order on only $63 \%$ of the trials. One interpretation of this finding is that the mechanism that computes depth order from boundaryflow information depends on processes that compute surface flow occurring very close to the boundary. However, even this large gap condition produced a perception of depth relative to control conditions. An alternative interpretation is that as the width of the gap between boundary and texture on either side increases, the texture fields are less likely to be perceived as extending up to the line. The finding that subjects perceive displays like the one in Figure 1A as surfaces ordered in depth implies that the visual system effectively "fills in" missing texture in the homogenous gap between the moving line and texture to produce the perception of continuous surfaces. Either of two factors may interfere with surface propagation as the width of the gap between texture and line is increased. First, the visual system may be less willing to propagate a surface across a homogenous space as the size of this gap is increased. For instance, the propagation of a surface from texture to an identically moving line (Figure 1) is an instance of the Gestalt law of common fate, which is likely to have limitations on its effective range of operation (Wertheimer, 1923). Second, if the texture field is sufficiently dense, its border may be perceived as a depth edge and thus disrupt the propagation of an apparent surface. In this case, disruption of surface propagation may be more likely as the width of the gap is increased.

Experiment 3 was designed to determine the factors that account for the weaker depth effects obtained when the distance between texture and boundary is increased. We examined the effects of gap size on perceived depth for displays that differed in the information specifying continuous surfaces extending up to the central boundary. In the objective contour conditions, shown in Figure 1A, a vertical line in the center of the display served as a boundary. Displays in this condition required the visual system to perform both surface propagation across a homogenous space and to compute the relative depth of surfaces. Figure 1B depicts the displays in the subjective contour conditions. In these displays, the horizontal lines used to generate the subjective contour extended through the texture to the contour and thus provided support for the perception of a single continuous surface.

By covarying gap size and the presence or absence of perceptual support for a continuous surface, it is possible to determine whether (1) surface-propagation processes or (2) processes that compute relative depth are responsible for the reduced effectiveness of boundary-flow displays as the retinal distance between boundary and texture is increased. Logically, either or both of these processes might be limited to local comparisons between boundary and texture. If surface propagation, but not the computation of relative depth, operates more effectively over small retinal distances, then we would expect objective contour displays to produce significantly weaker depth effects as a function of gap width. In this case, increasing gap width may not disrupt the perception of depth from subjective contour displays, since these displays in effect perform surface propagation for the visual system. If, on the other hand, processes involved in the computation of depth order are limited to local comparisons between boundary and texture, then both objective contour displays and subjective contour displays should produce significantly weaker depth effects as a function of increasing gap width.

In Experiment 3, subjects were also presented with displays containing texture regions of high, medium, and low density. This manipulation provided a test of the hypothesis that when gap size is large, a depth edge may be perceived at the border of texture fields and thus disrupt the propagation of a surface extending from the texture fields to the central boundary. This hypothesis predicts an interaction between gap size and density for displays in the objective contour condition. Small gap objective contour displays ought to be equally effective in generating the perception of depth at an edge, regardless of density. In the large gap objective contour conditions, however, low density displays may be more effective than high density displays, since they are less likely to generate the perception of a depth edge at the border of the texture field.

In previous studies of boundary flow, researchers have not attempted to control for the effects of fixation. It is possible that successive fixations to regions on either side of the boundary contribute to the obtained depth effects, particularly in large gap conditions. In Experiment 3, we attempted to minimize the effects of fixation by using brief presentations of the displays and instructing subjects to fixate the moving contour.

\section{Method}

Subjects. Seventeen unpaid adults, naive about the purpose of the experiment, served as subjects.

Apparatus. Each subject's stationpoint was fixed by a headrest. In all other aspects, the apparatus was identical to that used in Experiment 2.

Displays. Fourteen display conditions were presented. In each condition, a vertical contour was displayed at the center of the screen. Randomly distributed dots to the left and right of the contour formed two texture fields. All the dots in a texture field moved in synchronous horizontal motion across the screen at $1.6 \% \mathrm{sec}$. Texture on the left and right traveled simultaneously in opposite directions for $1 \mathrm{sec}$, and then reversed direction. Thus, the two texture fields alternately approached and receded from one another. On each trial, the lateral motion of the vertical contour at the center of the screen was identical to that of one of the two texture fields.

The display conditions differed in the sort of information they provided to specify the central contour. In the seven objective contour conditions, a vertical line served as a contour (see Figure 1A). Seven subjective contour conditions contained a subjective contour as an edge representation. This subjective contour was created by 10 horizontal lines, as in Experiments 2 and 3 (Figure 1B).

Within both of these contour conditions, the gap between texture field and contour was varied to produce small and large gap 
conditions. In the small gap conditions, the width of the unchanging gap between contour and identically moving texture on one side of the contour was $1.6^{\circ}$; the varying width of the gap between contour and texture on the other side ranged from $.1^{\circ}$ to $3.3^{\circ}$. In the large gap conditions, constant and varying gap sizes were $8.1^{\circ}$ and $6.5^{\circ}-9.7^{\circ}$, respectively.

The average dot density of texture fields was also varied to produce low, medium, and high density conditions. The numbers of dots per square degree of visual angle were $.05, .25$, and 1.25 , respectively. The visual angle of each dot was $.1^{\circ}$.

Two no-texture control conditions were also presented. In the objective contour control condition, a single vertical line moved alternately left and right across a dark homogenous background. The subjective contour control condition was identical to that used in Experiments 1 and 2.

Procedure. The general procedure was roughly equivalent to that used in Experiments 1 and 2, except that each display was presented for a brief time and an attempt to control subjects' fixations was made. Trials consisted of a 3-sec presentation of a centrally located point, followed immediately by a 3-sec presentation of the kinetic display. The subjects were instructed to fixate the centrally located point and then to fixate the translating vertical contour of the kinetic display for the duration of the trial. After each trial, the subject first pressed one of two keys on a keyboard to indicate whether he or she had actually fixated the contour for the duration of the trial. Next, the subject pressed one of three keys to indicate whether the left, the right, or neither side of the display was perceived as occluding the other side. As in Experiments 1 and 2, the subjects received practice trials with sheets of paper to ensure that they understood the instructions.

The subjects viewed a total of 140 trials, 10 from each display condition. As in Experiment 1, the order of presentation of display conditions was random. Total testing time was approximately $30 \mathrm{~min}$, including a short rest period in the middle of the experiment.

\section{Results and Discussion}

The comparatively small number of trials in which subjects responded that they had been unable to maintain fix- ation of the contour were not included in the data analysis. The results for the remaining trials are presented in Table 3. A one-way ANOVA carried out on the mean percentage of responses consistent with predicted depth order revealed a significant main effect for gap size $[F(1,16)=$ $15.98, p<.001]$ and a significant contour condition $\times$ gap size interaction $[F(1,16)=5.67, p<.03]$. Since the data clearly indicate that texture density was not related to the strength of the depth effect obtained, the means in Table 3 were collapsed for density. As expected, the subjective contour (small gap) condition and the objective contour (small gap) condition yielded strong depth effects, with $93.1 \%$ and $93.4 \%$ responding in the predicted direction, respectively. However, Tukey post hoc comparisons revealed differences in the effectiveness of the two large gap conditions. Observers responded in the predicted direction to $79.1 \%$ of the displays in the subjective contour (large gap) condition, a level of responding that did not differ significantly from either of the small gap conditions $(p>.05)$. In contrast, the level of predicted depth responding in the objective contour (large gap) condition, $68.7 \%$, was significantly lower than in both of the two small gap conditions $(p<.05)$. In addition, when the individual data were collapsed for density, 12 out of 15 subjects reported perceiving depth in the predicted direction more frequently in the subjective contour (large gap) condition than in the objective contour (large gap) condition. Three subjects responded with the predicted depth order more frequently in the objective contour (large gap) condition than in the subjective contour (large gap) condition. The 2 remaining subjects perceived the predicted depth order with equal frequencies in the two conditions. A sign test revealed that the number of subjects reporting the predicted depth order with greater frequency in

Table 3

Mean Percentages of Depth Order Judgments, with Standard Deviations: Experiment 3

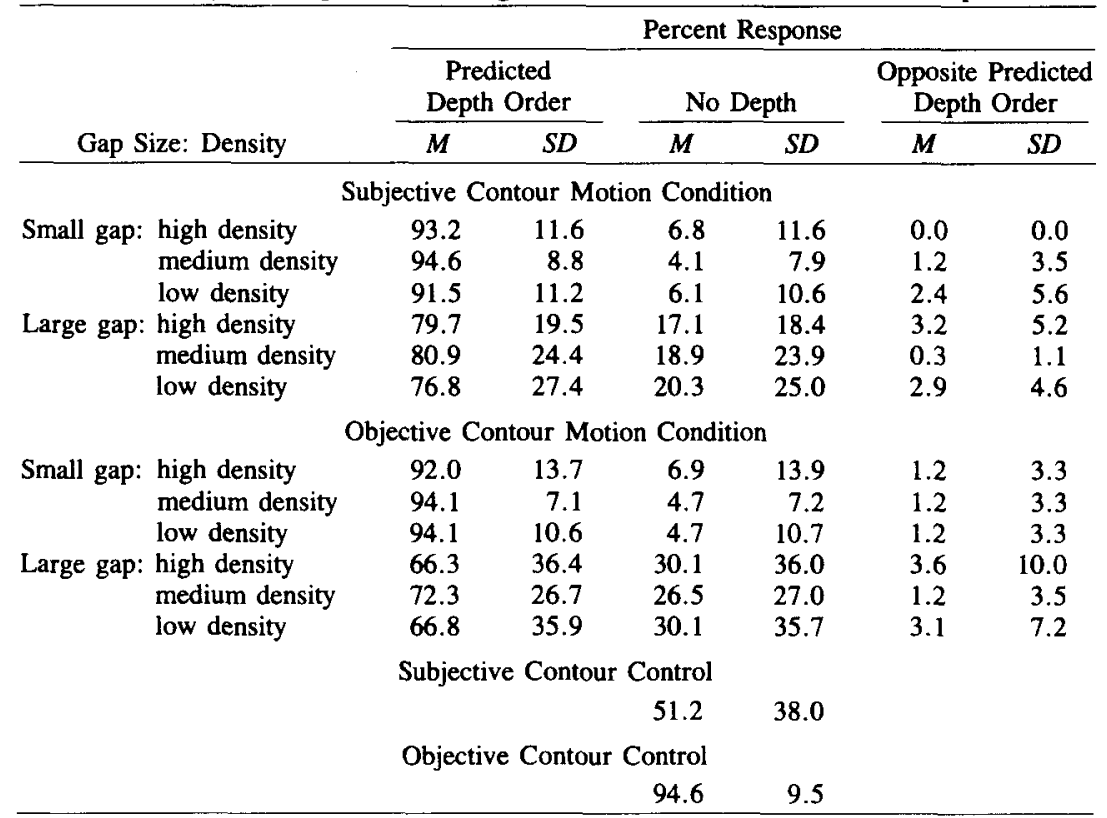


the subjective contour (large gap) condition than in the objective contour (large gap) condition was greater than chance $(p<.05)$.

The results of a follow-up experiment support the notion that the objective contour (large gap) displays are less likely to produce the perception of ordered depth than the other experimental conditions are. Eighteen naive adult subjects viewed a single continuous display, presented for $1 \mathrm{~min}$, of either the objective contour (large gap) condition or the subjective contour (large gap) condition. The procedure employed was similar to that used in Experiments 1 and 2, except that the practice trials were eliminated and subjects were simply asked to describe the display. Three of the 9 subjects viewing objective contour (large gap) displays for the first time spontaneously reported seeing one surface moving over another, with the predicted depth order. Three subjects reported the predicted depth effect only after being prompted after $30 \mathrm{sec}$ by the question, "Is there any depth suggested in the display?" The remaining 3 subjects failed to report the predicted effect: 2 of these subjects maintained that there was no apparent depth, and 1 subject reported depth in the opposite direction from that specified by boundaryflow information. In contrast, all 9 of the subjects viewing the subjective contour display reported the predicted depth effect: 7 did so spontaneously, and the remaining 2 reported depth only after prompting.

These results argue against the hypothesis that the mechanism which computes depth order from boundaryflow information operates only locally. Even displays in the objective contour (large gap) condition produced weak depth effects, as Yonas et al. (1987) reported for similar displays. The present finding that subjective contour (large gap) displays produce depth effects comparable to those obtained for small gap displays provides further evidence that the visual system is able to consistently use boundaryflow information to compute the depth order of surfaces when the retinal distance between boundary and projected surface texture is relatively large. This result is consistent with the hypothesis that perceptual support for continuous surfaces extending from boundary to texture strengthens the depth impression produced by boundaryflow displays with large gaps. The weaker depth effect obtained in the objective contour (large gap) condition as compared with the objective contour (small gap) displays suggests that these surface-propagation processes are disrupted for large separations between boundary and texture.

\section{GENERAL DISCUSSION}

In this article, we have attempted to continue and extend our investigation of human sensitivity to a recently discovered source of information for depth at an edge, the boundary-flow cue. Two components of boundaryflow information were distinguished: (1) An occluding surface projects surface texture that moves identically with the optical boundary projected by its edge; and (2) an oc- cluded surface projects surface texture that moves differently than the optical boundary projected by the edge of the surface that is occluding it. In Experiments 1 and 2, we examined the effectiveness of these two types of relations between boundary flow and surface flow as cues for the order of depth at an edge. A second distinction was made between a process that propagates a surface from projected surface texture to a boundary and one that computes relative depth from boundary-flow information. The local versus global processing characteristics of these two processes were examined in Experiment 3.

Overall, the results of the experiments reported here indicated the following: (1) Both the common motion and differential motion components of boundary-flow information generate the perception of depth at an edge when isolated in kinetic displays. (2) Differential motion of a boundary and texture may be a more effective cue for the order of depth at an edge than common motion of boundary and texture. (3) The perception of a stable depth order of surfaces from boundary-flow information does not require measurements of surface flow immediately adjacent to the boundary, provided there is perceptual support for the presence of surfaces extending up to the boundary. (4) Within the limits of the displays and procedures used in Experiment 3, the density of projected surface texture has no effect on performance.

These effects are not easily accommodated by the single existing algorithm for the recovery of relative depth from boundary-flow information (Thompson et al., 1985). The technique formalized by Thompson et al. involves a comparison of boundary flow with surface flow on either side of the boundary. This comparison process cannot serve as a model for depth perception from either the common motion or the differential motion displays used in this study, since these contain measurable flow on only one side of the boundary. By extension, the proposed model cannot account for the superiority of differential motion displays over common motion displays in generating perceived depth. A second characteristic of the Thompson et al. algorithm is that it is based on strictly local measurements of surface flow. The present results indicate that for translational motion at a constant depth, such local comparisons may be employed but are not necessary for the perception of relative depth by human beings.

A simple threshold-criterion model can account for depth effects produced when surface flow on only one side of a boundary is present. A surface-flow value differing from boundary flow by an amount below a threshold may be interpreted by the visual system as specifying an occluding surface, whereas a difference between boundary flow and surface flow that is above threshold would specify an occluded surface. Alternatively, the visual system may employ a comparison process when there is measurable surface flow on both sides of the boundary, and call upon an independent "default" process if surface flow on only one side is available. Future studies in which the velocities of texture fields and boundary are varied may help to determine whether a comparison of 
boundary flow and surface flow on both sides of the boundary is ever used by the visual system. When both texture fields have velocities that differ from the velocity of the boundary, the texture whose velocity differs least may be perceived as an occluding surface.

The stronger depth effect obtained for displays containing differential motion between boundary and texture compared to displays with common motion between boundary and texture was unexpected. One explanation for this effect is that the visual system propagates optical flow from regions in which flow is detected to regions where it is not. If this is the case, then there would be a bias toward perceiving untextured regions as having the same motion as nearby moving elements. Thus, an untextured region near the moving contour in boundary-flow displays would tend to share common motion with the contour and thus appear as foreground. Such a bias would enhance the perception of relative depth from differential motion displays, since boundary-flow information also specifies in this case that the untextured region is a foreground surface. In contrast, this bias would interfere with the perception of depth order from common motion displays, since boundary-flow information specifies in this case that the untextured region is background.

The reports of the subjects and the phenomenal impressions of the authors suggest that reliable computation of depth from boundary-flow information occurs only when the scene is experienced as being composed of two continuous surfaces. The data from Experiment 3 indicate that the perception of relative depth occurs consistently when the distance between projected texture and boundary is small, but becomes less consistent when this distance is increased. The results point to the conclusion that for large separations of texture and boundary (visual angle: constant gap, $8.1^{\circ}$; varying gap, $6.5^{\circ}-9.7^{\circ}$ ), whether the scene appears as continuous surfaces ordered in depth depends on (1) whether there is perceptual support (in this study, horizontal lines) for the presence of surfaces extending up to the boundary, and (2) to some extent, on the mental set of the subject. Increasing gap width significantly diminished the frequency of predicted depth judgments when perceptual support for surfaces was absent. However, this effect was most evident in the descriptions of large gap boundary-flow displays by naive subjects, suggesting that the experience that subjects in Experiment 3 had with the practice trials and/or the small gap displays created a mental set for interpreting the objective contour (large gap) displays as being composed of two continuous surfaces. Indeed, subjects with extensive experience (e.g., the authors) with boundary-flow displays are able to see objective contour displays as surfaces ordered in depth when the gaps between boundary and texture are even larger than those in the displays presented in Experiment 3.

One implication of the present findings concerns shape recognition for objects undergoing dynamic occlusion. Consider the case of a disk that is continuously covered and uncovered by a foreground surface (Figure 3). Such
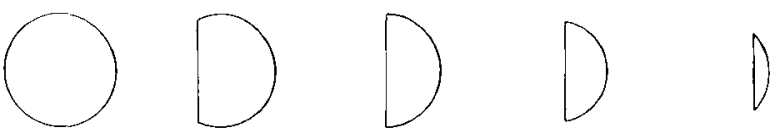

Figure 3. Schematic drawing of successive frames from the "screening effect" display created by Michotte, Thines, and Crabbe (1964). Subjects perceive a disk that is being covered and uncovered by a surface whose boundary is specified by the rectilinear portion of the figure.

occlusion makes recognition of the invariant form of the disk nontrivial, since it introduces a spurious edge at the occlusion boundary and the visual system must somehow make a distinction between the real edges of the disk and this spurious edge. Nakayama, Shimojo, and Silverman (1987) have shown that adults use stereoscopic depth to distinguish the intrinsic and extrinsic contours of a partially occluded object in a scene. We propose that, in the case of kinetic occlusion, depth from boundary-flow information can provide the basis for this classification of contours and make shape recognition possible.

Evidence for this can be found in Michotte's demonstration of the "screening effect" (Michotte, Thines, \& Crabbe, 1964), in which the progressive "blacking out" of a complete circular disk presented against a dark homogenous background creates the impression of an unchanging disk's being covered and uncovered by a second surface (see Figure 3). Michotte et al. maintained that Gestalt laws of perceptual organization explain this effect, by completing and giving phenomenal permanence to the disk. While we cannot rule out the "good form" of the disk as a factor in this case, note that boundaryflow information alone may explain the effect. The differential motion of the disk itself and the boundary defined by the rectilinear portion of the disk specifies that the boundary does not belong to the disk; it is, instead, the edge of an occluding surface. The results of Experiments 1 and 2 provide the first evidence that adults perceive depth at an edge on the basis of the differential motion of a boundary and the image of a surface without the apparently occluded surface's having "good form" and in the absence of kinetic stimulus information from the common motion of boundary and texture.

The surface-propagation process explored in this paper suggests a more general problem that must be solved by the visual system: Given a set of discrete elements lying on different, spatially separated surfaces, how do we determine where to fit smooth surfaces and where to postulate surface boundaries? Random-dot displays like the ones used in the present experiments typically generate the perception of continuous surfaces sprinkled intermittently with dots. That is, motion is attributed to the intervening regions between dots. In natural perception, we are able to perceive the spatial layout of surfaces that are sparsely textured or viewed under conditions of low illumination. This process of reconstructing a smooth surface between the discrete places where the surface is specified has been 
referred to as surface interpolation (Stevens, 1987). Although workers in computational vision have begun to explore this problem (e.g., Grimson, 1981), mechanisms of interpolation in human vision have not been studied intensively by psychophysicists.

A recent neural network model of human brightness perception proposed by Grossberg and Todorović (1988) suggests how static surface-interpolation processes might function. Grossberg and Todorović base their model on the interaction of boundary-detection processes and contrast-sensitive processes that "fill in" between the established boundaries. A recent model of motion perception developed by Marshall (1989) has shown how a simulated neural network can organize itself to become capable of propagating motion signals to nearby locations. This work might be extended to model our results through the positing of three interacting processes: (1) boundary detection; (2) surface interpolation based on the propagation of motion signals; and (3) computation of depth order of surfaces on the basis of the relative motion of projected surface texture and boundary.

\section{REFERENCES}

Andersen, G. J., \& Braunstein, M. L. (1983). Dynamic occlusion in the perception of rotation in depth. Perception \& Psychophysics, 34, 356-362.

BiNford, T. O. (1981). Inferring surfaces from images. Artificial Intelligence, 17, 205-244.

Craton, L. G., Yonas, A. (1988). Infants' sensitivity to boundary flow information for depth at an edge. Child Development, 59, 1522-1529.

Gibson, J. J., Kaplan, G. A., Reynolds, H. N., JR., \& Wheeler, K. (1969). The change from visible to invisible: A study of optical transitions. Perception \& Psychophysics, 5, 113-116.

Granrud, C. E., Yonas, A., Smith, I. M., Arterberry, M. E., Glicksman, M. L., \& Sorknes, A. C. (1984). Infants' sensitivity to accretion and deletion of texture as information for depth at an edge. Child Development, 55, 1630-1636.
GRIMson, W. E. L. (1981). From images to surfaces: A computational study of the human early visual system. Cambridge, MA: MIT Press.

GrossBerG, S., \& Todorović, D. (1988). Neural dynamics of 1-D and 2-D brightness perception: A unified model of classical and recent phenomena. Perception \& Psychophysics, 43, 241-277.

KAPLAN, G. A. (1969). Kinetic disruption of optical texture: The perception of depth at an edge. Perception \& Psychophysics, 6, 193-198.

MARSHALL, J. A. (1989). Neural networks for computational vision: Motion segmentation and stereo vision. Unpublished doctoral dissertation, Boston University.

Michotte, A., Thines, G., \& Crabbe, G. (1964). Les complements amodaux des structures perceptives (Studia psychologia). Louvain, Belgium: Publications Universitaires de Louvain.

Nakayama, K., Shimojo, S., \& Silverman, G. H. (1987). Investigative Ophthalmology \& Visual Science, 28(3, Supplement), 365.

REgAN, D. (1986). Visual processing of four kinds of relative motion. Vision Research, 26, 127-145.

STEVENS, K. A. (1987). Visual object perception from a computational perspective. In G. W. Humphreys \& M. J. Riddoch (Eds.), Visual object processing: A cognitive neuropsychological approach (pp. 1742). London: Erlbaum.

Thompson, W. B., Mutch, K. B., \& Berzins, V. A. (1985). Dynamic occlusion analysis in optical flow fields. IEEE Transactions on Pattern Analysis and Machine Intelligence, PAMI-7(2), 374-383.

Yonas, A., Craton, L. G., Thompson, W. B. (1987). Relative motion: Kinetic information for the order of depth at an edge. Perception \& Psychophysics, 41, 53-59.

Wertheimer, M. (1923). Principles of perceptual organization. In D. Beardslee \& M. Wertheimer (Eds.), Readings in perception (pp. 115-135). Princeton, NJ: Van Nostrand.

\section{NOTE}

1. We have referred to this depth information elsewhere as the relative motion cue. Unfortunately, this term signifies different things to different vision researchers. For instance, Regan (1986) discusses "four kinds of relative motion," and some colleagues have confused this cue with motion parallax. In this paper, we use "boundary flow" to refer to the information about relative depth order available at dynamic occlusion boundaries. This term has the merit of explicit reference to the boundarythat is, to the retinal projection of a depth edge between two surfaces.

(Manuscript received October 17, 1988; revision accepted for publication August 16, 1989.) 